\title{
EDITORIAL
}

\section{Phase Equilibrium Challenges and Modern Practice}

\author{
Roumiana P. Stateva*
}

Institute of Chemical Engineering, Bulgarian Academy of Sciences, 1113 Sofia, Bulgaria

Thermodynamic modeling and phase equilibria are at the heart of chemical process design. For example, (70 to 90) \% of the equipment and energy costs in modern chemical plants are related to separation and purification processes and they are designed largely on the basis of thermodynamic equilibrium. More importantly, new chemical engineering processes often involve multicomponent, non-ideal system, which can exhibit a variety of possible phase equilibria. Furthermore, the processes are often performed at pressures and temperatures near the critical point or near the phase boundary, which further complicates the solution of the phase equilibrium problem. Last, but not least, phase equilibria calculations are executed nowadays not only as stand-alone calculations, but also as a part of a more general simulation, synthesis or design case, performed by a process simulator, where they are run thousands of times. In these cases the reliability and robustness of phase equilibria calculations is of utmost importance since their failure at any stage will disrupt completely the solution process.

This Special Issue is focused on current techniques and today's approaches employed to solve robustly, efficiently and effectively challenging phase equilibria problems in process design and simulation. The issue includes 6 contributions. Each one of them has a link to the philosophy and practice of predicting, interpreting, modeling, and calculating phase equilibria of complex systems in a broad range of applications.

Property estimation and data regression are an important element of the development of applied thermodynamic models. Paster, Brauner and Shacham in their contribution present a refinement of the TRC-QSPR method, originally advocated by Shacham and colleagues for prediction of phase equilibrium related properties, focused at accurate prediction of the vapor pressure-temperature relation for a target compound. The method is a valuable tool as at present, vapor pressure data, which are essential for phase equilibrium computation, process and product design, are available only for a small fraction of the compounds of interest to the chemical industry.

Marcilla, Reyes-Labarta, Serrano Cayuelas and Olaya discuss data regression from a different perspective - namely phase equilibrium data regression in order to obtain the appropriate parameters for any thermodynamic model to be

*Address correspondence to this author at the Institute of Chemical Engineering, Bulgarian Academy of Sciences, 1113 Sofia, Bulgaria; Tel: + 359297934 81; E-mail: thermod@bas.bg used in separation equipment design for chemical process simulation and optimization. They summarize and analyze their experience from previous work on the capabilities and limitations of the existing $\mathrm{G}^{\mathrm{E}}$ models for the regression of condensed phase equilibrium data in ternary systems. The authors advocate strategies that can be included in the correlation algorithms to improve their convergence characteristics and avoid inconsistencies.

The drive to use green benign solvents as an alternative to hazardous organic solvents in such important applications as recovery of high added value substances from natural matrices, remediation of soil, etc. has focused attention to the application of hot pressurized water (HPW). The design and modeling of a HPW extraction process requires, however, a detailed knowledge of the solubility of the solid solutes in the solvent. In their contribution Fornari and co-authors discuss and compare the predictive capabilities, advantages and deficiencies of the UNIFAC-based models and approaches based on Regular Solution Theory to represent the solubility of an important class of compounds, namely polycyclic aromatic hydrocarbons, in subcritical water.

The next contribution presented by Polishuk and colleagues addresses a very important question in phase equilibria calculations, namely: Are an advanced theoretical basis and numerical complexity of an EoS sufficient to guarantee success in predicting the experimental thermodynamic property data? The authors perform a profound analysis of the capabilities of the most sophisticated SAFT, namely SAFT-VR-Mie, and reveal earlier unnoticed numerical pitfalls in its modeling capacity for even simple spherical non-polar molecules such as methane.

Ávila-Méndez, Justo-García, García-Sánchez, and García-Flores compare the capabilities of PC-SAFT, yet another SAFT representative, with those of the PengRobinson cubic EoS to predict the multiphase behavior of a model mixture of methane, carbon dioxide, hydrogen sulfide, and water. Mixtures of water with other components with similar properties, like polar components or compounds with hydrogen bonding, are of great importance in the oil extraction industry, and their reliable modeling is a severe test to any thermodynamic model and numerical technique.

Zhang, Bonilla-Petriciolet and Rangaiah, in their excellent extensive review, examine the state-of-the art of the current global optimization methods and their applications for modeling the phase behavior of systems with and without chemical reactions including the prediction of azeotropes and critical points. The authors consider selected 
deterministic and stochastic optimization algorithms, review their use for phase stability analysis, Gibbs free energy minimization and parameter estimation in phase equilibrium models, and conclude that despite the many advances in this area, research in global optimization for phase equilibrium modeling and calculations will continue to be an active field in chemical engineering.
Finally, I would like to say that it has been my honor and pleasure to guest-edit this issue, and that I am, of course, most grateful to the colleagues from around the world for their outstanding contributions which made this special issue a reality.

(C) Roumiana P. Stateva; Licensee Bentham Open.

This is an open access article licensed under the terms of the Creative Commons Attribution Non-Commercial License

(http://creativecommons.org/licenses/_by-nc/3.0/) which permits unrestricted, non-commercial use, distribution and reproduction in any medium, provided the work is properly cited. 